\title{
The gunshot-related injuries in trauma (GRIT) study: A profile of patients affected by gunshot- related orthopaedic injuries across South Africa
}

\author{
J Masters, ${ }^{{ }^{*}}$ DPhil, MRCS; M Laubscher, ${ }^{2 *}$ FCOrth (SA), MMed (Orth); S Graham, ${ }^{2,3}$ MB ChB, MRCS, MSc (Res), FRCS (Tr\&Ortho); \\ L Marais, ${ }^{4} \mathrm{MB}$ ChB, FCOrth (SA), MMed (Orth), PhD; N Ferreira, ${ }^{5}$ BSc, MB ChB, HDip Orth, FCOrth (SA), MMed (Orth), PhD; \\ M Held, ${ }^{2} \mathrm{MD}$, PhD, FCOrth (SA); J Viljoen, ${ }^{6}$ FCOrth (SA); T Pillay, ${ }^{7}$ MB BCh; S Maqungo, ${ }^{2}$ MB ChB, FCOrth (SA), MMed (Orth), \\ PGDip HPE; M Costa, ${ }^{1}$ PhD, FRCS (Tr\&Ortho); on behalf of the Orthopaedic Research Collaboration for Africa (ORCA) investigators \\ ${ }^{1}$ Oxford Trauma, Nuffield Department of Orthopaedics, Rheumatology and Musculoskeletal Sciences, University of Oxford, UK \\ ${ }^{2}$ Orthopaedic Research Unit, Department of Orthopaedic Surgery, Faculty of Health Sciences, Groote Schuur Hospital and University of Cape Town, \\ South Africa \\ ${ }^{3}$ Department of Clinical Sciences, Liverpool School of Tropical Medicine, Liverpool, UK \\ ${ }^{4}$ Department of Orthopaedic Surgery, Faculty of Health Sciences, University of KwaZulu-Natal, Durban, South Africa \\ ${ }^{5}$ Division of Orthopaedics, Department of Surgical Sciences, Faculty of Medicine and Health Sciences, Stellenbosch University, Cape Town, \\ South Africa \\ ${ }^{6}$ Department of Orthopaedic Surgery, Faculty of Health Sciences, University of Pretoria, South Africa \\ ${ }^{7}$ Department of Orthopaedic Surgery, Faculty of Health Sciences, University of the Witwatersrand, Johannesburg, South Africa \\ ${ }^{*}$ Joint first authors.
}

Corresponding author: J Masters (james.masters@ndorms.ox.ac.uk)

\begin{abstract}
Background. South Africa (SA) has one of the highest gun-related mortality rates in the world -20 people per day. The available data, however, do not reflect the substantial number of patients suffering non-lethal firearm injuries. Gunshot-related injury has been recognised as a highly costly healthcare problem by individual treating centres in SA and other countries; however, no 'national picture' has been examined in detail.

Objectives. To explore the burden of gunshot-related orthopaedic injuries across SA.

Methods. A multicentre research network was established in SA, and 37 orthopaedic units across 9 provinces participated. A prospective, observational cohort study was performed during a 2-week period in 2019. Patients were screened, enrolled and reported by local orthopaedic teams. Patients were included if they had at least one acute gunshot-related orthopaedic fracture referred to the orthopaedic service. Patients were asked additional questions around baseline health-related quality-of-life (HRQOL) and personal circumstances. Follow-up was at 8 weeks after injury.

Results. Thirty-seven centres enrolled 135 patients over the 2-week study period. Western Cape Province had the highest number of reported cases $(n=52 ; 39 \%)$, followed by Gauteng $(n=35 ; 26 \%)$ and KwaZulu-Natal $(n=29 ; 21 \%)$. The median age of patients was 30.5 years and the majority were male (89\%). Forty-three percent of patients had been either shot or stabbed prior to this injury. Fifty-two percent of all patients required fracture fixation surgery and $11 \%$ required wound debridement without fracture fixation. HRQOL data were collected successfully at baseline, but follow-up data were available for $<25 \%$ of cases.

Conclusions. Gunshot-related orthopaedic injuries represent a significant burden of disease in the SA healthcare environment. This study highlights several areas for further research in the management of the injuries and associated outcomes.
\end{abstract}

S Afr Med J 2021;111(7):655-660. https://doi.org/10.7196/SAMJ.2021.v111i7.15236

Gunshot-related injury in the civilian setting is a complex problem with several important variables determining incidence, injury pattern and outcome. Worldwide, these injuries are focused in countries with a high level of gun ownership in combination with particular socioeconomic circumstances. ${ }^{[1]}$ The legacy of these injuries for those who survive possibly varies by injury severity, treatment availability and social setting. The data around these outcomes are rarely available, particularly in low- and middle-income countries, where the burden of gun violence is high. ${ }^{[1]}$

In South Africa (SA), the burden of gun violence is among the highest in the world. ${ }^{[2]}$ Gun-related mortality is estimated at 20 per day (11th highest globally). ${ }^{[3]}$ Data around non-fatal gun injures are more difficult to come by, particularly as there is no infrastructure for surveillance. However, single-centre data identify a significant burden of work for public hospitals and cost to the state healthcare provider. ${ }^{[4]}$

Given the multidimensional nature of gunshot-related trauma, the circumstances of violence, its socioeconomic drivers and the consequences must be considered. ${ }^{[5]}$ However, more information is needed on the geographical spread of violence and the populations involved.

Current data on gun violence in SA focus on mortality. ${ }^{[3,6]}$ There is a lack of understanding regarding the burden to individuals, society and health funders created by gun violence. Important questions also remain around best practice and variation in care pathways. There is no national guidance and each clinical unit is required to manage 
patients according to local guidelines. Most of the evidence available is generated in high-income countries and the military. This may be limited in its relevance to middle-income settings such as SA. ${ }^{[7]}$

Given the important current limitations to the understanding of the burden of gunshot-related orthopaedic injuries across the country, we conducted a multicentre observational cohort study with the following objectives:

- to determine the number of people affected by gunshot orthopaedic injuries at the participating centres

- to understand the anatomical distribution of injuries and associated surgical treatment strategies

- to understand the demographics of the people affected by gunshotrelated violence

- to review whether patients are alive or dead at 8 weeks, to monitor the percentage who attend routine follow-up and to explore collection of follow-up data in a 'difficult to follow-up' population, including health-related quality of life (HRQOL).

\section{Methods \\ Setting}

This study was set across the 9 provinces of SA, a middle-income country with a population of 56 million. ${ }^{[8]}$ Site investigators were recruited from the South African Orthopaedic Association that represents orthopaedic surgeons across the country.

\section{Study design}

A prospectively collected observational cohort study was conducted. The total study period was from 18 February to 26 April 2019. Patient identification and recruitment was open for 2 weeks, from 18 February to 4 March 2019. The 2-week recruitment period was based on previous studies using similar embedded clinical teams to recruit patients and return data. ${ }^{[9]}$

After this, there was 8 weeks' follow-up to record patient outcomes according to usual clinical care in this setting. Each site investigation team monitored orthopaedic trauma admissions at their centre for patients who met the inclusion criteria mentioned below. Only routinely collected anonymised medical data were captured at baseline and follow-up. Where patients lacked capacity, consent was sought to enrol in the study and ask specific research questions.

The study population comprised adults $\geq 18$ years old, with at least one gunshot-related orthopaedic fracture to limb, spine or pelvis.

The primary outcomes were as follows:

- to determine the number of people affected by gunshot-related orthopaedic injuries at the participating centres

- to determine the distribution of injuries and associated surgical treatment strategies.

The secondary outcomes were as follows:

- to determine the social and demographic patient characteristics at baseline

- to determine whether alive or dead at discharge or 8 weeks

- to determine attendance at follow-up - an embedded feasibility component.

Adequate follow-up is a well-recognised challenge in this setting. Associated with the secondary objectives was the capture of HRQOL. This was captured at baseline, asking for pre-injury state and followup. EuroQol 5 dimension 5 level (EQ-5D-5L), a tool for measuring health status across 5 domains (pain, anxiety/depression, mobility, self-care and usual activities) was used. ${ }^{[10]}$ Each domain was scored on a scale from 1 (no problems) to 5 (extreme problems). There was an associated visual analogue scale, where 0 represents the worst health state imaginable and 100 represents perfect health.

\section{Data capture method and data fields}

Each investigator team identified all eligible patients presenting to their unit during the study window period. Eligible patients were approached, and their consent sought to answer research-specific questions related to the study EQ-5D-5L, education and housing data. Where patients lacked capacity or did not want to provide answers to research questions, only anonymous routinely collected data were recorded. All data were recorded on the REDCap (Research Electronic Data Capture) online secure platform. All centres were approached and asked to confirm that no cases were missed at their unit.

\section{Statistical analysis}

No formal hypothesis testing was undertaken. Descriptive summary statistics were used to outline the relevant metrics. Each parameter is presented, where appropriate, with an associated expression of uncertainty, e.g. 95\% confidence interval. EQ-5D-5L scores are represented descriptively using summary statistics for the visual analogue scale (VAS), and raw scores reported for the 5 domains. No health utility scores were calculated in the absence of an SA reference population dataset. Analysis and graphic generation were undertaken using the in situ software on REDCap (v8.4.3), STATA 15.1 (StataCorp., USA) or Google maps.

\section{Ethical approval}

Ethical approval was given by the University of Cape Town (UCT) Human Research Ethics Committee (ref. no. HREC 755/2018). The study was conducted in line with the principles of the Declaration of Helsinki. Each unit used UCT approval or sought reciprocal approval from the relevant health board. The study was registered prospectively on the Clinical Trials Registry (ref. no. NCT03854591). Additional institutional permission was sought where requested.

\section{Results}

Public hospitals across SA took part in the study (37 of 52 centres with orthopaedic specialist care). All centres were invited to participate. Of the 15 centres that did not participate, 7 did not respond at all, 2 declined owing to lack of capacity and 6 did not participate, as they were not able to obtain the necessary permission in time.

Fig. 1 outlines the location of the participating centres.

\section{Burden of injury}

A total of 135 unique cases of gunshot-related fractures was captured across 8 of SA's 9 provinces. Table 1 outlines the distribution of cases across the country, representing all of the eligible patients seen at the participating institutions.

Western Cape Province had the highest number of cases $(n=52$; $39 \%)$, followed by Gauteng $(n=35 ; 26 \%)$ and KwaZulu-Natal $(n=29$; $21 \%$ ). There was significant variation in the number of patients seen at each centre in each province. The highest volume of work was undertaken in the larger city hospitals with large urban catchment areas (Supplementary Table 1: http://samj.org.za/public/sup/15236-1.pdf).

\section{Injury profile and treatment}

Table 2 outlines the anatomical pattern of injury and reported treatment. The femur was most commonly affected $(n=29)$ and mostly treated surgically. The next most commonly affected regions were also in the lower limb. Injuries to the leg (tibia/fibula) were much less commonly managed with surgery $(28.2 \%)$. 


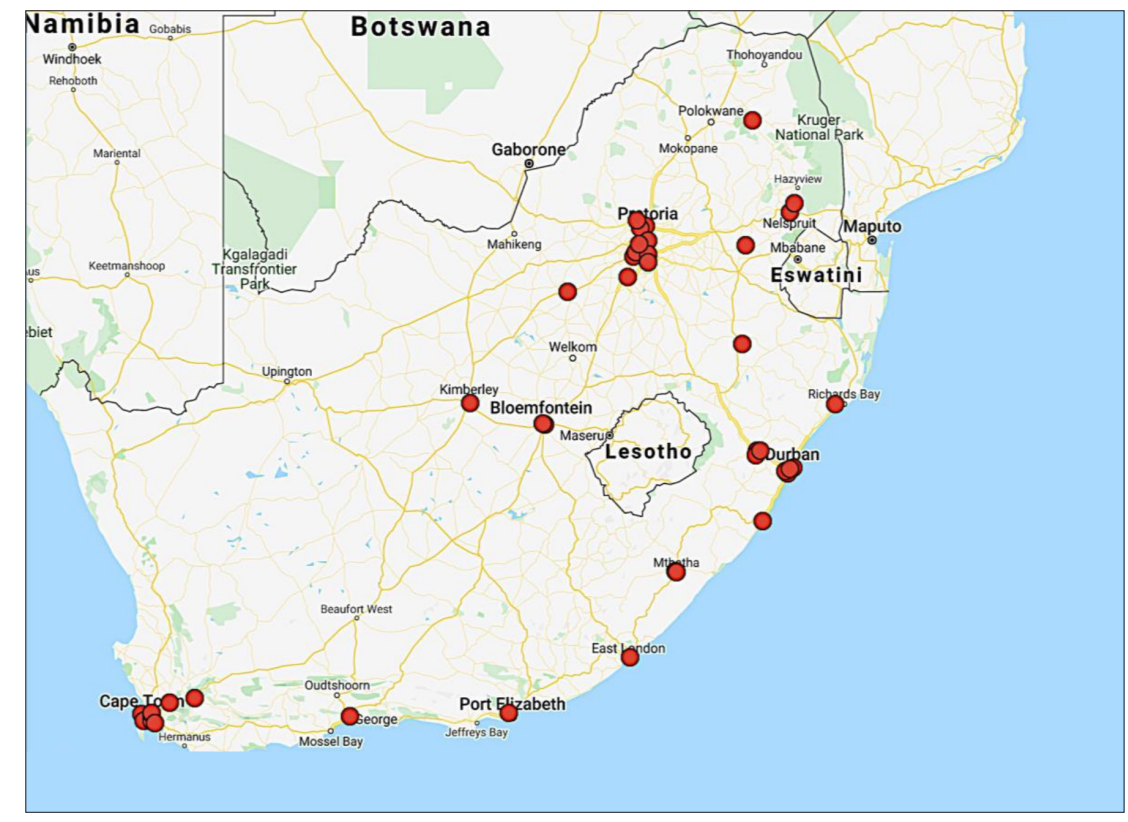

Fig. 1. Geographical representation of centres participating in the gunshot-related injuries in trauma (GRIT) study.

\section{Patient profile}

Patients affected by gun violence are overwhelmingly young (mean age 32.5 years) males (89\%) (Table 3). A significant proportion is either unemployed or employed in low-skilled occupations. There are also a very high number of cases $(n=51)$ where the individual had been either stabbed or shot prior to the episode captured in this study. Of the 76 patients who disclosed their HIV status, $16(21 \%)$ reported their status as positive.

\section{Patient outcomes and follow-up}

Fewer than $20 \%$ of patients identified returned for follow-up (Table 4). In most cases, this was due to the patients not attending routine clinical follow-up. For those with a recorded outcome, over half were discharged home from inpatient hospital care by 8 weeks.

\section{Health-related quality of life}

Table 5 outlines the mean VAS scores in the EQ-5D-5L outcome tool. The average score

\section{Table 1. Cases by province, 18 February - 4 March 2019}

\begin{tabular}{llll}
\hline Province & Gunshot-related injuries, $\boldsymbol{n}(\%)$ & Population, $\boldsymbol{n}^{*}$ & $\begin{array}{l}\text { Study centres/orthopaedic units } \\
\text { per province, } \boldsymbol{n}\end{array}$ \\
\hline Western Cape & $52(39)$ & 6300000 & $10 / 10$ \\
Gauteng & $35(26)$ & 13400000 & $6 / 14$ \\
KwaZulu-Natal & $29(21)$ & 11100000 & $11 / 13$ \\
Free State & $8(6)$ & 2800000 & $2 / 4$ \\
Mpumalanga & $6(4)$ & 4300000 & $2 / 3$ \\
Eastern Cape & $5(4)$ & 7000000 & $3 / 3$ \\
Limpopo & $0(0)$ & 5404868 & $1 / 3$ \\
North West & $0(0)$ & 3700000 & $1 / 1$ \\
Northern Cape & $0(0)$ & 1200000 & $1 / 1$ \\
Total & $135(100)$ & 55600000 & $37 / 52$ \\
${ }^{*}$ Population data are based on the 2016 community survey ${ }^{[12]}$ & &
\end{tabular}

Table 2. Profile of injury and treatment

\begin{tabular}{|c|c|c|c|c|c|c|}
\hline Area injured & $\begin{array}{l}\text { Delayed fixation of } \\
\text { fracture }(>72 h), n(\%)\end{array}$ & No surgery, $\boldsymbol{n}(\%)$ & $\begin{array}{l}\text { Non-orthopaedic } \\
\text { surgery, } n(\%)\end{array}$ & $\begin{array}{l}\text { Wound debridement } \\
\text { only, } n(\%)\end{array}$ & $\begin{array}{l}\text { Wound } \\
\text { debridement } \\
\text { and orthopaedic } \\
\text { surgery, } n(\%)\end{array}$ & Total, $n$ \\
\hline Shoulder & $1(20)$ & $4(80)$ & 0 & 0 & 0 & 5 \\
\hline Arm/humerus & $1(12.5)$ & $1(12.5)$ & $1(12.5)$ & $1(12.5)$ & $4(50)$ & 8 \\
\hline Elbow & 0 & $3(75)$ & 0 & 0 & $1(25)$ & 4 \\
\hline Forearm & $1(14)$ & $1(14)$ & 0 & $2(28)$ & $3(43)$ & 7 \\
\hline Hand/wrist & $2(22)$ & $2(22)$ & 0 & $3(33)$ & $2(22)$ & 9 \\
\hline Spine & $1(17)$ & $3(50)$ & $2(33)$ & 0 & 0 & 6 \\
\hline Pelvis & 0 & $3(100)$ & 0 & 0 & 0 & 3 \\
\hline Hip & $1(50)$ & $1(50)$ & 0 & 0 & 0 & 2 \\
\hline Femur & $12(41)$ & $2(7)$ & 0 & 0 & $15(52)$ & 29 \\
\hline Knee & $1(14)$ & $2(28)$ & 0 & $3(43)$ & $1(14)$ & 7 \\
\hline Leg & $4(17)$ & $11(46)$ & $1(4)$ & $1(4)$ & $7(29)$ & 24 \\
\hline Foot and ankle & $2(14)$ & $6(43)$ & 0 & $3(21)$ & $3(21)$ & 14 \\
\hline Total & $26(22)$ & $39(33)$ & $4(3)$ & $13(11)$ & $36(31)$ & 118 \\
\hline
\end{tabular}




\begin{tabular}{|c|c|}
\hline Variable & $n(\%)^{*}$ \\
\hline Age (years), mean (median) (IQR), $n=135$ & $32.5(30.5)(25-38)$ \\
\hline Male, $n=135$ & $122(89.7)$ \\
\hline Patient is aware of HIV status, $n=135$ & $76(63.3)$ \\
\hline HIV-negative ${ }^{\dagger}$ & $60(79)^{\ddagger}$ \\
\hline HIV-positive ${ }^{\dagger}$ & $16\left(21 ; ;^{\ddagger} 11.9^{\varsigma}\right)$ \\
\hline \multicolumn{2}{|l|}{ Education, $n=119$} \\
\hline Unschooled & $3(2.5)$ \\
\hline Primary & $42(35.3)$ \\
\hline Secondary & $64(53.8)$ \\
\hline Higher & $10(8.4)$ \\
\hline \multicolumn{2}{|l|}{ Employment, $n=119$} \\
\hline Unemployed & $43(36.1)$ \\
\hline Unskilled & $45(37.8)$ \\
\hline Skilled & $12(10.1)$ \\
\hline Professional & $2(1.7)$ \\
\hline Previous arrest, $n=114$ & $44(38.6)$ \\
\hline Previous stab or gunshot wound, $n=119$ & $51(42.9)$ \\
\hline Police aware of incident, $n=119$ & $79(66.4)$ \\
\hline Household members, median (IQR), $n=118$ & $4(3-6)$ \\
\hline Rooms per household, median (IQR), ${ }^{9} n=117$ & $3(2-4)$ \\
\hline \multicolumn{2}{|c|}{ 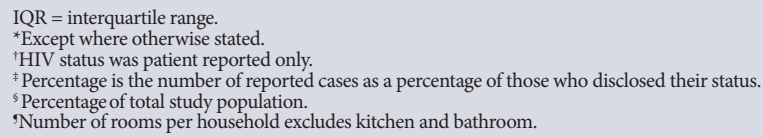 } \\
\hline
\end{tabular}

\begin{tabular}{ll}
\multicolumn{2}{l}{ Table 4. Follow-up and primary outcome at $\mathbf{8}$ weeks } \\
\hline Variable & $\boldsymbol{n}(\%)$ \\
\hline Attendance at follow-up, $n=135$ & $25(18.5)$ \\
Yes & $53(39.3)$ \\
No & $13(9.6)$ \\
No appointment given & $44(32.5)$ \\
Missing & \\
Outcome of hospital stay, $n=135$ & $2(1.5)$ \\
Dead & $51(37.8)$ \\
Missing & $82(60.7)$ \\
Alive & $75(55.6)$ \\
Discharged & $5(3.7)$ \\
Transferred to alternative institution & $1(1.2)$ \\
Absconded & $1(1.2)$ \\
Ongoing inpatient care &
\end{tabular}

Table 5. Summary of health-related quality-of-life scores Baseline* health-related quality-of-life EQ-5D VAS $(n=114)$, mean (SD)

$78.1(22.0)$

Follow-up health-related quality-of-life EQ-5D VAS ( $n=25)$, mean (SD)

$69.1(16.4)$

$\mathrm{EQ}-5 \mathrm{D}=$ EuroQol- 5 dimensions; $\mathrm{VAS}=$ visual analogue scale; $\mathrm{SD}=$ standard deviation . *Baseline refers to pre-injury health-related quality of life.

after injury was lower (69.1) than before injury (78.1). Assessment of the scores of those with both baseline and follow-up data, showed that most patients did not recover their health at 8 weeks (Fig. 2).

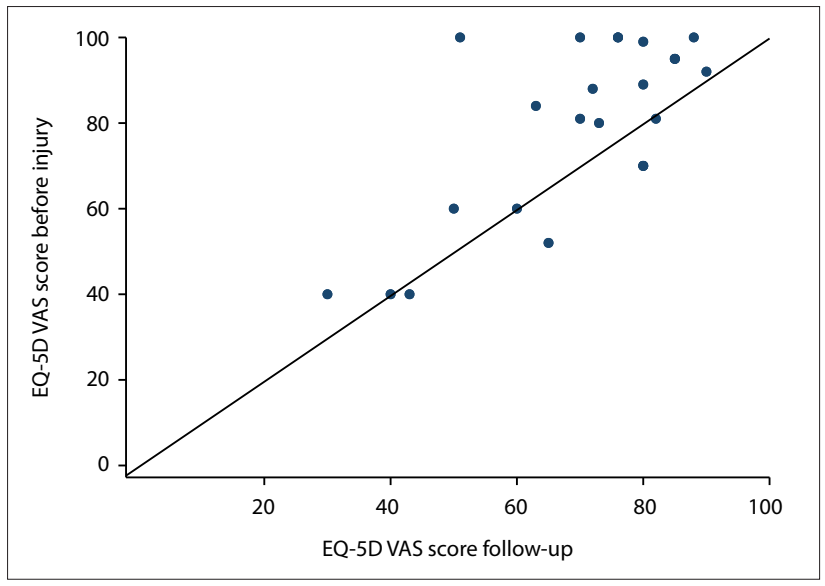

Fig. 2. Patients with baseline and follow-up data for health-related quality of life. The plotted line represents an equal visual analogue scale score before injury and at follow-up, i.e. recovery to the baseline health state. Note that the follow-up rate is low (25\%), which limits the extrapolation of the findings to the overall study population. $(E Q-5 D=$ EuroQol-5 dimensions; VAS $=$ visual analogue scale.)

The scores for each domain of health indicate that these injuries affect not only typical musculoskeletal elements, such as mobility, but also anxiety (Supplementary Table 2: http://samj.org.za/public/ sup/15236-2.pdf).

\section{Discussion}

This is the first multicentre study of gunshot-related injury in SA, which captured data across varied urban and rural environments. Most units participating have no research support and patients were recruited and data collected by the clinical team. Therefore, we selected a short recruitment period based on previous studies with a similar methodology. ${ }^{[9]}$ Most injuries were centred on the major conurbations of the Western Province, Gauteng and KwaZulu-Natal. A total of 135 patients was identified over a 14-day study period at participating centres - a rate of $>9$ patients per day. It should be noted that only orthopaedic units at public hospitals participated. Around $90 \%$ of the population is dependent on public healthcare, while the remaining $10 \%$ access private healthcare.$^{[11]}$ Also, in rural SA, primary orthopaedic care is administered by general practitioners. It is therefore likely that the numbers presented underestimate the number of gunshot-related fractures in SA.

The femur was the most commonly affected anatomical region. A range of surgical treatment strategies was employed, depending on the location of the gunshot injury. That two-thirds of the patients captured in this study undergo some type of surgery, is of considerable concern for frontline healthcare providers and health authorities. In a previous study, the average cost of inpatient care for one of these episodes was estimated at USD2 940. ${ }^{[4]}$ If the 2 weeks captured here were repeated for a calendar year, then this could account for >USD10 million per year in SA. These costs have been found in other studies of gunshot patients. ${ }^{[12]}$ Furthermore, they only consider the hospital costs and do not identify human resources of a multidisciplinary team, post-discharge costs and broader societal costs, such as inability to return to work.

Bibliometric analysis of gunshot-related injuries highlights that most of the literature around treatment is from the USA. ${ }^{[7]}$ The treatment paradigm in the USA and in other high-income countries is for almost all patients to undergo surgical treatment. ${ }^{[13,14]}$ These treatment algorithms are not necessarily relevant to low-velocity 
civilian gunshot injuries. A significant percentage of patients did not receive surgical treatment. These cases included incomplete fractures that did not require surgical fixation, but also certain complete fractures, where non-operative treatment in the form of casting or bracing was used. In most cases where fractures did not require surgical fixation, wound washout or debridement was not performed. The local non-operative treatment approach seen in this study and possibly practised more widely in SA is not based on high-level evidence, but on local protocols developed iteratively over time. Such practice may be a pragmatic solution to the overwhelming volume of work. Clearly, an understanding of outcome for each strategy is imperative to determine the relative benefit of each. This is a key future research objective.

The demographic characteristics of the patients in this study can be described as typical of the broader SA population compared with those in the General Household Survey data 2018. ${ }^{[15]}$ When considering the level of educational attainment, $40 \%$ completed only primary education and $73.9 \%$ were either not employed or in low-skilled employment. Most patients were young males $<35$ years of age.

Almost half of the patients had previous experience with interpersonal violence. These data raise the question whether an intervention at the time of hospitalisation can prevent future episodes. Programmes around early intervention in interpersonal violence have been used in both the UK and USA. ${ }^{[5,16]}$ The Cure Violence (formerly Cease Fire) programme uses 'social levers' to reduce the incidence of retaliatory shooting and associated murder by up to $41 \% \cdot{ }^{[17]}$ One of the central tenets of the Cure Violence programme is the targeting of high-risk individuals. Given that $>40 \%$ of the patients captured in this study had previously been either stabbed or shot, they can clearly be described as high risk.

Patients in these socioeconomic brackets may be highly vulnerable to 'health shock events' ${ }^{[18]}$ Therefore, the effect of these injuries may further impoverish the members of society who can least afford it. ${ }^{[19]}$

The data identify that the police were only aware of $67 \%$ of the gunshots captured in this study. For policymakers, this has implications for the national understanding of gunshot-related crime, which is largely based on police data.

Most patients were discharged after their inpatient hospital care episode. The EQ-5D-5L tool for HRQOL was captured at baseline and at follow-up. The data suggest that the majority of patients with HRQOL do not recover within the first 8 weeks. However, the poor return to follow-up makes any definitive conclusions impossible. Given the known significant impact such injuries may cause, it is possible to speculate that the tail of recovery is long and significant for patients with these injuries.

This is the first multicentre study across SA that surveyed gunshotrelated orthopaedic injury. It involves a range of settings, including large urban areas and rural hospitals, which adds significantly to the external validity of the results. These data are an important first look at the problem on a national scale.

While all attempts were made to include as many centres as possible, not all facilities providing healthcare to patients suffering gunshot-related injuries were included. Therefore, it remains difficult to estimate the true scope of the healthcare burden - most likely this is still underestimated. A further consideration is that the patients were identified through a specialty-specific network (orthopaedics); there might have been relevant numbers of injuries referred to other specialties. Furthermore, hospitals in rural settings have no specialist units and orthopaedic injuries are treated by general practitioners.
The follow-up return rate was low, and the results must be interpreted in this context. This is a phenomenon of the study population, who typically have a low return to follow-up for routine care. ${ }^{[20,21]}$ The need of patients to allocate resources of time and money for follow-up may present a very high barrier to ongoing engagement. Other studies have improved follow-up rates by compensating patients for their return hospital visits ${ }^{[22]}$ or modifying the follow-up process itself. ${ }^{[23]}$ The latter approach may be more challenging in orthopaedic trauma care, where critical outcomes such as infection in the presence of metalwork or nonunion require extensive periods of follow-up.

\section{Conclusions}

The abovementioned data highlight that the people affected by gunrelated injuries across SA are largely in low-skilled employment and have previously been victims of interpersonal violence. Although a small sampling window, there is possibly a spectrum of treatment offered that may be determined by injury severity, pattern and variation in practice. This contrasts with the treatment discussed in the literature from high-income and military settings. The variation observed may be explained by clinical factors, such as pattern of injury and soft-tissue coverage; however, it may equally be explained by access to expertise or appropriate facilities.

This significant public health problem for SA and these data identify several key areas for further research in the management of the injuries and associated outcomes. In addition to public health and social measures, there is a pressing need for observational and interventional studies. This may include a registry approach or mandating gunshot injuries as notifiable.

Future studies should capture patient-reported outcome measures, help define variations in clinical practice and complications of care. Such work will need to be appropriately resourced to assist in overcoming some of the significant barriers identified in this study, in particular those relating to patient follow-up. Future work must engage all stakeholders to deliver benefit for SA patients but also contribute insights to other countries where similar problems are faced, such as Central and South America.

Declaration. None.

Acknowledgements. The study team would like to acknowledge support from the Newton International/Research Councils UK (RCUK) as part of the University of Oxford/University of Cape Town PhD exchange programme, as well the South African Orthopaedic Association. We are grateful to Annemie Stewart for help with database design.

Author contributions. JM: designed the study, co-ordinated the collaborative investigators, wrote the manuscript and interpreted the data; ML: designed the study, co-ordinated the collaborative investigators and wrote the manuscript; SG: conceived the study and provided critical revisions to the manuscript; LM: oversaw regional study participation (KwaZuluNatal) and provided critical revisions to the manuscript; NF: oversaw regional study participation (Western Cape) and provided critical revisions to the manuscript; $\mathrm{MH}$ : supervised the study design and provided critical revisions to the manuscript; JV: oversaw regional study participation (Pretoria) and provided critical revisions to the manuscript; TP: oversaw regional study participation (Johannesburg) and provided critical revisions to the manuscript; SM: oversaw regional study participation (Western Cape) and supervised the study design and manuscript production; and MC: supervised the study design and manuscript production. 
Funding. This study was supported by the Newton International/RCUK University of Oxford/University of Cape Town PhD exchange programme and the South African Orthopaedic Association.

Conflicts of interest. NF reports grants from Orthofix SRL, personal fees from Orthofix SRL and personal fees from Smith \& Nephew, all of which are outside the submitted work; MC is a National Institute for Health Research (NIHR) senior investigator. The views expressed in this article are those of the author(s) and not necessarily those of the NIHR, or the Department of Health and Social Care. The University of Oxford received research grant funding from the NIHR, EU, RCS England and industry.

1. Karp A. Estimating Global Civilian-Held Firearms Numbers. Geneva: Global Small Arms Survey Graduate Institute of International and Development Studies, 2017

2. Keegan M. The Proliferation of Firearms in South Africa, 1994 - 2004. Johannesburg: Gun Free South Africa, 2005.

3. Global Burden of Disease Injury Collaborators, Naghavi M, Marczak LB, et al. Global mortality from firearms, 1990 - 2016. JAMA 2018;320(8):792-814. https://doi.org/10.1001/jama.2018.10060

4. Martin C, Thiart G, McCollum G, Roche S, Maqungo S. The burden of gunshot injuries on orthopaedic healthcare resources in South Africa S Afr Med I 2017:107(7):626-630. https://doi.org/10.7196/ SAMJ.2017.v107i7.12257

5. Butts JA, Roman CG, Bostwick L, Porter JR. Cure violence: A public health model to reduce gun violence. Butts JA, Roman CG, Bostwick L, Porter /R. Cure violence: A public health model to reduce gun violence.
Ann Rev Pub Health 2015;36(1):39-53. https://doi.org/10.1146/annurev-publhealth-031914-122509

6. Meel B. Twenty-three-year trend in firearm deaths in the Transkei subregion of South Africa (1993 2015). Med Sci Law 2018;58(2):102-108. https://doi.org/10.1177/0025802418758802

2015). Med Sci Law 2018;58(2):102-108. https://doi.org/ $10.1177 / 0025802418758802$
7. Held M, Engelmann E, Dunn R, et al. Gunshot induced injuries in orthopaedic trauma research. A bibliometric analysis of the most influential literature. Orthop Traumatol Surg Res 2017;103(5):801-807. https://doi.org/10.1016/j.otsr.2017.05.002

8. World Health Organization. Country profile: South Africa. https://www.who.int/countries/zaf/en/ (accessed 17 May 2021)
9. Spence RT, Panieri E, Rayne SL. A multicentre evaluation of emergency abdominal surgery in South Africa: Results from the GlobalSurg-1 South Africa study. S Afr Med J 2016;106(2):163-168. https://doi. org/10.7196/SAMJ.2016.v106i2.10183

10. Herdman M, Gudex C, Lloyd A, et al. Development and preliminary testing of the new five-level version of EQ-5D (EQ-5D-5L). Qual Life Res 2011;20(10):1727-1736. https://doi.org/10.1007/s11136-011-9903-x

11. Dell AJ, Gray S, Fraser R, Held M, Dunn R. Orthopaedic surgeon density in South Africa. World J Surg 2018;42(12):3849-3855. https://doi.org/10.1007/s00268-018-4709-4

12. Eriksson A, Norberg J, Nilsson T, Hardcastle T. The costs of a bullet - inpatient costs of firearm injuries in South Africa. S Afr Med J 2009;99(6):442-444.

13. Abghari M, Monroy A, Schubl S, Davidovitch R, Egol K. Outcomes following low-energy civilian gunshot wound trauma to the lower extremities: Results of a standard protocol at an urban trauma center. lowa Orthop J 2015;35:65-69.

14. Sathiyakumar V, Thakore RV, Stinner DJ, Obremskey WT, Ficke JR, Sethi MK. Gunshot-induced fractures of the extremities: A review of antibiotic and debridement practices. Curr Rev Musc Med 2015;8(3):276-289. https://doi.org/10.1007/s12178-015-9284-9

15. Statistics South Africa. General Household Survey. Pretoria: StatsSA, 2018.

16. Williams DJ, Currie D, Linden W, Donnelly PD. Addressing gang-related violence in Glasgow: A preliminary pragmatic quasi-experimental evaluation of the community initiative to reduce violence A preliminary pragmatic quasi-experimental evaluation of the community initiative to
(CIRV). Agg Violt Behav 2014;19(6):686-691. https://doi.org/10.1016/j.avb.2014.09.011

17. National Institute for Justice. Evaluation of ceasefire - Chicago. 2009. http://www.skogan.org/files/ Evaluation_of_CeaseFire-Chicago_Main_Report.03-2009.pdf (accessed 17 May 2021).

18. Alam K, Mahal A. Economic impacts of health shocks on households in low and middle income countries: 8. Alam K, Mahal A. Economic impacts of health shocks on households in low and middle incon
A review of the literature. Glob Health 2014;10:21. https://doi.org/10.1186/1744-8603-10-21

19. Leive A, Xu K. Coping with out-of-pocket health payments: Empirical evidence from 15 African countries. Bull World Health Organ 2008;86(11):849-856. https://doi.org/10.2471/blt.07.049403

20. Ologunde R, Rufai SR. Surgical follow-up in low-income and middle-income countries. Lancet Glob Health 2013;1(3):el32. https://doi.org/10.1016/S2214-109X(13)70061-5

21. Ologunde R, Maruthappu M, Shanmugarajah K, Shalhoub J. Surgical care in low and middle-income countries: Burden and barriers. Int J Surg 2014;12(8):858-863. https://doi.org/10.1016/i.ijsu.2014.07.009 22. Graham SM, Harrison WJ, Lalloo DG, et al. HOST Study - HIV in Orthopaedic Skeletal Trauma Study: Protocol for a multicentre case-cohort study. S Afr Orthop J 2018;17(3):53-58.

23. Congdon N, Yan X, Lansingh V, et al. Assessment of cataract surgical outcomes in settings where follow-up is poor: PRECOG, a multicentre observational study. Lancet Glob Health 2013;1(1):e37-e45. https://doi. org $/ 10.1016 / \mathrm{s} 2214-109 \mathrm{x}(13) 70003-2$

Accepted 20 January 2021 